\title{
Controlling an Automated Wheelchair via Joystick/Head-Joystick Supported by Smart Driving Assistance
}

\author{
Thomas Röfer and Christian Mandel and Tim Laue
}

\begin{abstract}
With this work, we encourage the application of smart driving assistance algorithms to support the operator of an automated wheelchair in complex navigational situations. On the basis of an empirical study in which eight untrained subjects performed a given course using a conventional joystick and a proportional head-joystick respectively, we are able to prove benefits resulting from the application of a newly developed driving assistance module. Altering the translational and rotational velocities in situations where an obstacle blocks the usercommanded way, the driving assistance module significantly improves driver-performance by preventing all collisions along the way.
\end{abstract}

\section{INTRODUCTION}

Automated wheelchairs that are equipped with sensors and a data processing unit constitute a special class of wheeled mobile robots, termed smart wheelchairs in general literature overviews [1], [2]. Beside general scientific fields of work, such as autonomous navigation approaches, or mapping and self-localization algorithms, the shared spatial reference system between the operator and the smart wheelchair gives rise to certain issues related to user interfaces and shared control problems. For instance, Simpson et al. showed in [3], [4] how to combine discrete driving commands coming from voice control, with navigation assistance provided by reactive navigation approaches.

The work presented in this paper is an extension of research we conducted earlier [5]. In comparison to our original work, progress has been made in two main aspects: the new wheelchair used is equipped with a differential drive in contrast to an Ackermann steering in our previous system. This led to a completely different description of the possible motions of the wheelchair and the safety areas that are the basis for the assistance module presented. The other aspect is that by using laser range sensors rather than sonar sensors it was possible to keep the full usability of the original wheelchair intact, i.e. the additional equipment we installed is not in the way. Thereby, we avoided one of the main

This work has partly been funded by the European Commission in the context of the 6th Framework Programme for RTD in the context of the project "SHARE-it - Supported Human Autonomy for Recovery and Enhancement of cognitive and motor abilities using information technologies" under the contract number FP6-045088 and by the Deutsche Forschungsgemeinschaft in the context of the SFB/TR 8 "Spatial Cognition" research center.

T. Röfer and T. Laue are with Deutsches Forschungszentrum für Künstliche Intelligenz $\mathrm{GmbH}$, Safe and Secure Cognitive Systems, Enrique-Schmidt-Str. 5, 28359 Bremen, Germany \{thomas.roefer, tim.laue\}@dfki.de

C. Mandel is with the Department of Mathematics and Computer Science - FB3, University of Bremen, PO Box 330440, 28334 Bremen, Germany cmandelduni-bremen. de

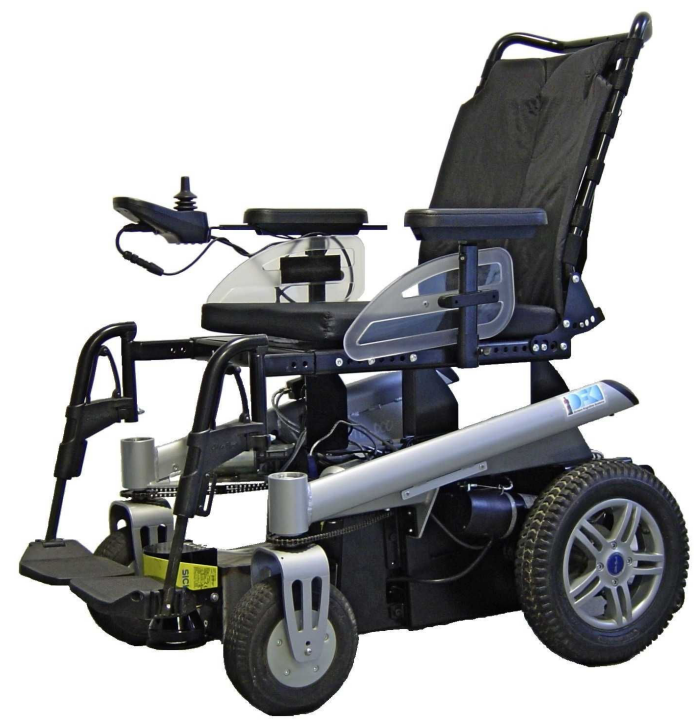

Fig. 1. The Xeno by Otto Bock Healthcare. One of the laser range sensors can be seen behind the foot rests.

drawbacks most research prototypes of smart wheelchairs seem to have.

The paper is structured as follows: first, we give an overview of the automated wheelchair. Afterwards, the actual driving assistance module is presented. Since we tested it with two different input devices, a short review of special input devices follows. The experimental evaluation is described next. The paper closes with the conclusions and an outlook.

\section{SYSTEM OVERVIEW}

The experimental platform is based on the power wheelchair Xeno by the German manufacturer Otto Bock Healthcare. The wheelchair has its drive wheels in the back and castor wheels in the front. The specialty of that wheelchair model is the active castor wheels. They are always rotated by motors to the orientation that matches the current driving direction. Hence, all the problems that normally occur with passive castor wheels in wheelchairs, such as blocking wheels after a change of the driving direction, are solved in this model. The wheelchair offers a CANBUS interface that allows wire-tapping between the joystick and the motor control.

The Xeno was extended by two laser range sensors (model S300 by Sick), one in the front behind the foot rests, the other one in the back. They measure the distance to the closest obstacles in a height of $12 \mathrm{~cm}$ above the ground and have 
an opening angle of $270^{\circ}$. The drive wheels were equipped with wheel encoders with a resolution of approximately $2 \mathrm{~mm}$ driving distance per tick. A micro controller board is counting the encoder ticks. The micro controller, a USBCANBUS adapter, and both laser range sensors (via USBRS422 converters) are connected to a USB hub. A netbook class PC is controlling the system through a single USB cable connected to the hub.

\section{Driving Assistance}

The basic idea of the driving assistance module (the Driving Assistant) is to detour obstacles in a way that is most likely to be acceptable for the user. By taking into account the desired travelling direction in terms of the curve indicated by the user via the joystick or another device that gives similar directions, the assistant decides whether to avoid an obstacle, and if yes, to which side, i. e. to the right or to the left. The Driving Assistant controls both speed and steering of the wheelchair. The speed is always reduced in a way that the wheelchair cannot collide with the obstacles in the environment. The steering is controlled to avoid obstacles to the side the user intends to, or not to avoid an obstacle if the user directly heads toward it. In the latter case, the wheelchair would simply stop to prevent a collision.

In the following we illustrate how this behavior is achieved. We will first describe the representation of the environment used, the local obstacle map. Afterwards, the safety regions are presented that are used to search the map for obstacles. Finally, the obstacle avoidance itself is explained.

\section{A. Local Obstacle Map}

The laser range sensors cannot see the entire environment of the wheelchair. Beside the fact that they can only measure at a height of $12 \mathrm{~cm}$ above the ground, part of the environment is also hidden by the wheelchair's casing and by the wheels. Therefore, a local obstacle map is used to store readings of the environment. Thereby, the wheelchair not only knows about the obstacles that are currently visible, but also about those that have been perceived before when the wheelchair was at a different position.

The local obstacle map or occupancy grid [6] is a quadratic array of cells, each of which encodes the certainty about the presence of an obstacle at the corresponding location in the environment of the wheelchair (cf. Fig. 3). The position of the wheelchair relative to the map is static with respect to translational movements and dynamic with respect to rotational changes. This means that the wheelchair's $(x / y)$-position, i. e. the middle of the rear axle, constantly remains in the center of the map (within \pm 0.5 cells), whereas the orientation of the wheelchair relative to the map changes during operation. As a consequence of the wheelchair's static position at the center, the map has to be shifted according to the current translational movement. That movement is determined using the wheel encoders (i. e. odometry).

The local obstacle map covers an area of $7.5 \times 7.5 \mathrm{~m}^{2}$ around the wheelchair, i.e., the distance between the center

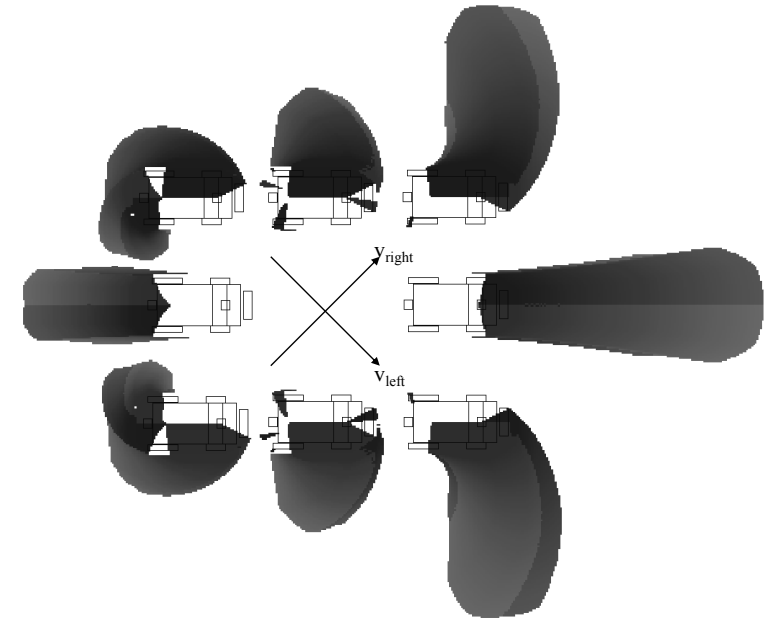

Fig. 2. Some examples of safety regions for different speeds of the left and right wheels

of the map and its border is approximately twice the length of the overall stopping distance of the wheelchair.

\section{B. Safety Regions}

The wheelchair has a rather complex shape and the shape of the area it "touches" during a braking maneuver is even more complex. To effectively avoid obstacles, the knowledge about this safety area is pertinent. The safety area depends on dynamic and static parameters. The dynamic parameters are the current translational and rotational driving speeds, and their expected change due to the commands previously sent to the wheelchair. The static parameters are the maximum errors expected in measuring the dynamic parameters, the shape of the actual wheelchair, the latency with which it executes commands, its deceleration during braking maneuvers, and the behavior of the steering while braking. In case of the Xeno, the latter is rather interesting, because the internal motor control of the wheelchair always turns back the active castor wheels to "straight ahead" when a full stop command is issued. Thereby, the Xeno breaks out of the curve it was previously driving while braking.

Since the computation of the safety area is rather complex, a large number of safety areas have been pre-computed and stored in a table. The index in this table is the direction of the vector that comprises of the dimensions left wheel speed and right wheel speed:

$$
\phi=\operatorname{atan} 2\left(v_{\text {right }}, v_{\text {left }}\right)
$$

Thereby, any combination of translational and rotational motion of the wheelchair can be represented, e. g., forwards, backwards, turning on the spot, curves, etc. (cf. Fig. 2). A safety region is represented as a set of cells, each of which the wheelchair will reach during braking. Each cell contains the minimum speed norm with which the wheelchair has to drive before braking so that the cell will still be reached during the braking maneuver. The speed norm is the sum of the absolute values of both wheel speeds:

$$
v_{\text {norm }}=\left|v_{\text {right }}\right|+\left|v_{\text {left }}\right|
$$




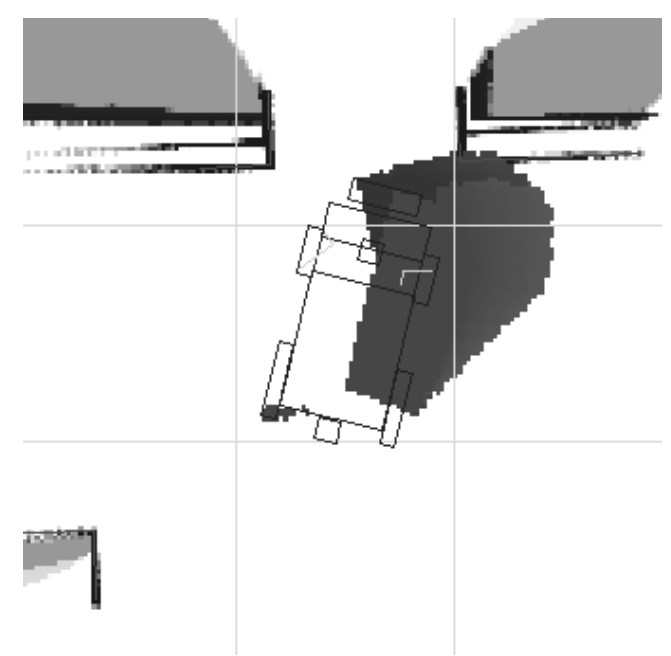

Fig. 3. Deciding on which side the user wants the obstacle to be passed. Obstacles within the brighter region are avoided to the left, while obstacles in the darker region are avoided to the right.

The speed norm represents how fast the wheelchair is moving, independent of the direction of the motion. Together, $v_{\text {norm }}$ and $\phi$ can be seen as a representation of the wheelchair's motion in polar coordinates, one of which describes the speed of motion and the other one describes the direction.

\section{Obstacle Avoidance}

A typical situation is depicted in Figure 3: The wheelchair is placed in front of a doorway. If it simply drove forwards, it would hit into the right door post. The user indicates a right turn (as shown by the region checked for obstacles). Therefore the Driving Assistant infers that he or she wants to pass the obstacle (i.e. the right door post) on the right-hand side. Thus it reinforces its steering command to the right. If, instead, the user would indicate a left curve, the assistive system assumes that the driver wants to pass the obstacle on the left-hand side, i. e., to pass through the doorway.

To determine the avoidance maneuver that matches the intention of the human driver best, the direction he or she indicates with the joystick is considered. All the time, the module searches the local map for obstacles based on this direction employing the corresponding safety region, i.e. the region that the wheelchair will reach within the next few seconds. If the wheelchair is already detouring, the direction indicated by the user may deviate from the system's current steering direction. Thus, the Driving Assistant always assesses the world from the user's point of view, and this view includes the judgment whether an obstacle is on the left or on the right side of the intended driving direction.

To allow the Driving Module to reconstruct this assessment, it was pre-processed for each cell in a safety region whether it is better to avoid an obstacle at the corresponding real-world position on the left side or on the right side. For instance, if the wheelchair would hit the obstacle with its front left, it would try to avoid it on the right side. In contrast, if a collision was expected on the left side
TABLE I

AVOIDANCE DIRECTIONS WHEN DRIVING FORWARDS

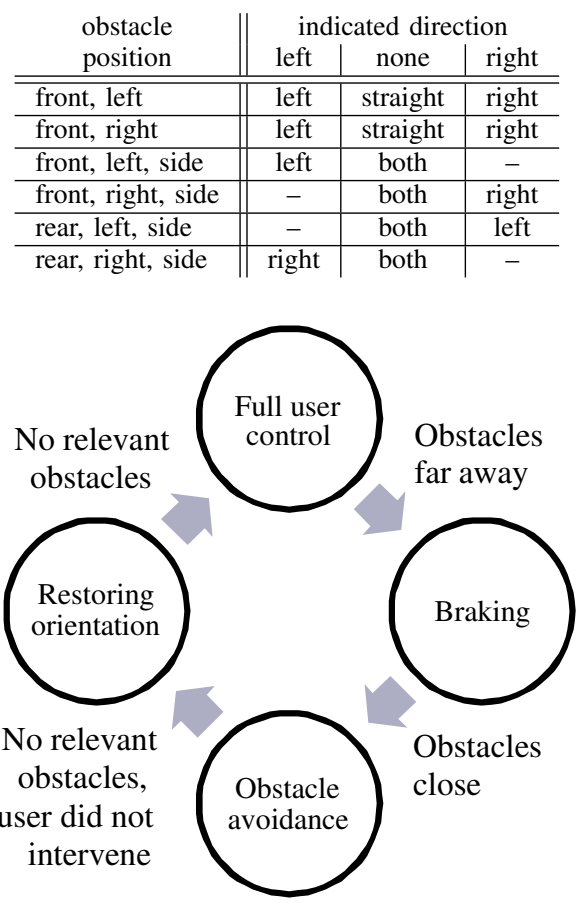

Fig. 4. The finite state machine of the Driving Assistant

behind the driving axle, the wheelchair would also turn to the left. So, the avoidance direction depends on the obstacle's position relative to the centre of the wheelchair's driving axle. The different cases are listed in Table I for forward movements and in Table II for backward motion. Using these decision tables, the safety regions can be enriched by the appropriate avoidance directions for each cell of the covered area. Figure 3 shows an example of a resulting decision diagram. Note that if the indicated course directly points towards an object, the Driving Assistant will not intervene at all. This is because in such a case it is likely that the driver's intention is to move close to that obstacle and not to pass around it, e.g. when docking to a desk. In contrast obstacles are always avoided to both sides if they are on the wheelchair's sides (i.e. next to it on the left or on the right), e.g., to make sure that the wheelchair does not get stuck between doorposts.

If an obstacle is avoided in open space, it can be confusing

TABLE II

AVOIDANCE DIRECTIONS WHEN DRIVING BACKWARDS

\begin{tabular}{l||c|c|c}
\multicolumn{1}{c||}{$\begin{array}{c}\text { obstacle } \\
\text { position }\end{array}$} & \multicolumn{3}{c}{ indicated direction } \\
left & none & right \\
\hline \hline rear, left & left & straight & right \\
\hline rear, right & left & straight & right \\
\hline rear, left, side & left & both & - \\
\hline rear, right, side & - & both & right \\
\hline front, left, side & - & both & left \\
\hline front, right, side & right & both & -
\end{tabular}


for the user, because the driving direction changes without an explicit user command. Therefore, the Driving Assistant remembers the driving direction when the avoidance maneuver begins. If the user does not react to the change in direction during the avoidance action, i. e., he or she does not move the joystick, the system will return to the original path indicated by the user after the obstacle was circumvented. Therefore, a finite state machine is maintained that controls the behavior of the wheelchair (cf. Fig. 4). As long as there are no obstacles, the user is in full control of the vehicle. If the obstacles are far away or the user does not wish to avoid them, only the speed of the wheelchair is reduced. The wheelchair will avoid an obstacle if it can determine an avoidance direction from the direction indicated by the user and the position of the obstacle(s). If the wheelchair passed the obstacle and the user did not intervene by moving the joystick, the Driving Assistant will restore the orientation of the wheelchair. In summary, it can be stated that the user always stays at least partially in control: the driving assistant will never drive faster than indicated by the user, and it will never change the driving direction (forward/backward). The transition to automated control is seamlessly. There are no notifications about interventions. From experiments with a simpler system, i.e. obstacles threatening the safety regions result in deceleration of wheel speeds, up to a full stop maneuver, we know that notifications about obstacles that result in a full stop of the system are sometimes desirable, and they will be integrated in the future.

\section{USER INTERFACES}

Traditional automated wheelchairs are operated by joystick, directly translating the user's hand movements into translational and rotational velocities. While such interfaces suit a large audience, certain disabilities may require appropriate alternatives, e.g. Brain-Computer-Interfaces [7], [8], head posture [9] or gaze [10] interpretation, and natural language communication [11], [12], to name but a few.

With regard to people needing specialized input devices due to handicaps such as tetraplegia, this study analyzes the impact of the proposed driving assistance module (cf. Sec. III) on a common hand-operated joystick, and a headjoystick (cf. Fig. 5), first described in [13], [14], [15]. While people are familiar with the use of the former device, i.e. the deflection of the joystick maps proportional onto the direction and the velocities of the wheelchair, the later one

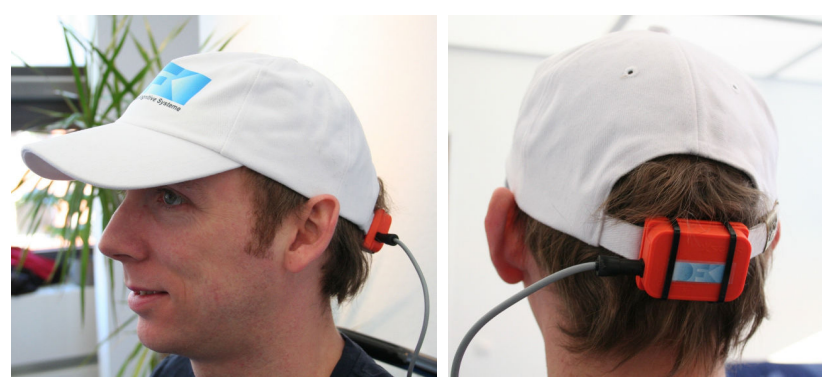

Fig. 5. The head-joystick connected to a cap is more sophisticated. The basic idea is to let the user of an automated wheelchair control the translational and rotational velocity by continuous pitch and roll movements of his/her head (cf. Fig. 6). Still able to observe the environment by turning the head around the free yaw-axis without causing any control commands, the user's head movements around the remaining two axes must exceed a so-called dead-zone in order to evoke a desired movement.

\section{EXPERIMENTAL EVALUATION}

\section{A. Subjects and Procedure}

Eight healthy subjects participated in this study. Because none of the participants had controlled the automated wheelchair Xeno before, a preparation phase of some five minutes preceded each test run. During this short training phase, the subjects had the chance to become familiar with both input devices. After that, the actual experiment started. In four consecutive test runs, the participants were asked to navigate the wheelchair safely but in a timely manner from their starting position located at the lower left of the maps depicted in Fig. 8(a)-8(d), to the upper right, and back again. In doing so each subject utilized the standard joystick and the head-joystick, with and without the driving assistance module respectively.

\section{B. Experimental Setup}

In order to compare and evaluate the results of the different experimental runs, a common frame of reference is needed. In our case, this is a global map of the environment, and the ability to localize the wheelchair at any point in time within the map.

For creating the map (shown in Fig. 8(a) - 8(d)), we used the GMapping implementation from OpenSLAM [16]. It is a highly efficient Rao-Blackwellized particle filter that learns grid maps from raw laser range and odometry data [17]. The

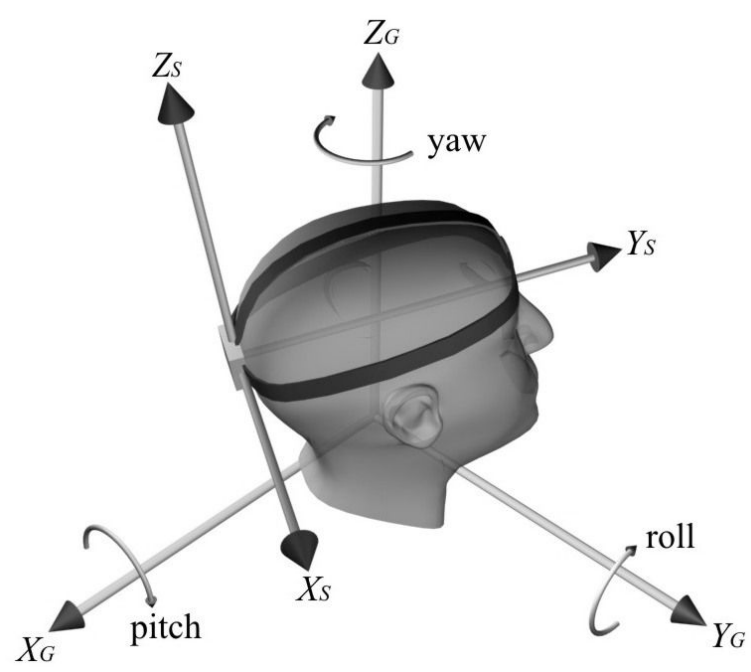

Fig. 6. First described in [13], [14], [15], the head-joystick is mounted at the back of the user's head and outputs the posture of the sensor's local coordinate system $S$ w.r.t. a fixed global coordinate system $G$. The pitchand roll-deflections are subsequently translated into steering commands. 
TABLE III

PERFORMANCE CRITERIA FOR SUBJECTS S1-S8 PERFORMING TEST RUNS I-IV:

TIME OF TRAVEL $t[s]$, DRIVEN DISTANCE $d[m]$, AND NUMBER OF COLLISIONS $c$.

\begin{tabular}{|c|c|c|c|c|c|c|c|c|c|c|c|c|}
\hline$\frac{\text { Criterion }}{\text { Subject }}$ & $t_{I}$ & $d_{I}$ & $c_{I}$ & $t_{I I}$ & $d_{I I}$ & $c_{I I}$ & $t_{I I I}$ & $d_{I I I}$ & $c_{I I I}$ & $t_{I V}$ & $d_{I V}$ & $c_{I V}$ \\
\hline S1 & 71.45 & 34.35 & 0 & 101.51 & 34.97 & 0 & 106.12 & 35.43 & 0 & 137.68 & 47.93 & 0 \\
\hline $\mathrm{S} 2$ & 78.98 & 37.26 & 1 & 106.10 & 36.20 & 0 & $50.09^{*}$ & $18.72^{*}$ & - & 131.14 & 43.71 & 0 \\
\hline S3 & 66.51 & 33.98 & 0 & 101.49 & 35.80 & 0 & 131.68 & 35.67 & 0 & 146.68 & 41.30 & 0 \\
\hline S4 & 77.57 & 35.18 & 0 & 132.97 & 40.42 & 0 & 113.24 & 35.60 & 0 & 135.47 & 38.73 & 0 \\
\hline S5 & 68.62 & 33.74 & 0 & 96.88 & 34.10 & 0 & 99.97 & 35.76 & 1 & 136.90 & 40.13 & 0 \\
\hline S6 & 70.99 & 34.00 & 0 & 90.66 & 35.36 & 0 & 116.69 & 34.05 & 1 & 119.60 & 37.72 & 0 \\
\hline S7 & 86.21 & 33.27 & 0 & 110.94 & 34.39 & 0 & 100.44 & 33.76 & 0 & 145.57 & 39.44 & 0 \\
\hline S8 & 78.05 & 32.23 & 1 & 108.51 & 34.88 & 0 & 90.24 & 32.02 & 1 & 140.72 & 46.63 & 0 \\
\hline
\end{tabular}

grid map used for our experiments has a cell size of $2 \times 2 \mathrm{~cm}^{2}$ and a total size of $11 \times 6 \mathrm{~m}^{2}$.

Real-time localization during the experimental runs has been realized by a modified version of the GMapping implementation. While leaving the map data untouched, a small number of particles keeps track of the wheelchair's position.

A further module that has been activated in order to keep the experiments comparable was the so-called SmoothMotionController. For experimental test runs with and without driving assistance this module limited maximum speeds and accelerations to a common base.

\section{Results}

Table III shows some performance criteria for the eight participants that performed the four test runs. It can be noticed that for both the input devices the average time of execution and the length of the traveled distance increased when performing with the driving assistance module. In particular the execution time of standard joystick test runs increased about $41.9 \%\left(\overline{t_{I}}=74.80 \mathrm{~s}, \overline{t_{I I}}=106.13 \mathrm{~s}\right)$, while head-joystick test runs increased about $35.3 \%\left(\overline{t_{I I I}}=\right.$ $\left.101.06 s, \overline{t_{I V}}=136.72 \mathrm{~s}\right)$ in execution time. The same trend can be found for the traveled distance. The length of the test runs performed with standard joystick increased about $4.42 \%$ $\left(\overline{d_{I}}=34.25 \mathrm{~m}, \overline{d_{I I}}=35.77 \mathrm{~m}\right)$, while head-joystick test runs increased about $28.6 \%\left(\overline{d_{I I I}}=36.63 \mathrm{~m}, \overline{d_{I V}}=41.95 \mathrm{~m}\right)$ in length of travel. The third performance criterion, the average number of collisions is 0 for both input devices supported by the driving assistance module, and $0.25 / 0.42$ for standard joystick and head-joystick without driving assistance. So without assistance, five collisions happened in 15 runs, i. e. in $33 \%$ of the tests. In addition, subject 2 aborted test run III at the point marked by the red $X$ in Fig. 8(c), since he felt very insecure with the head-joystick. However, he performed even better than the average when using the Driving Assistant.

\section{CONCLUSIONS AND FUTURE WORK}

The first important issue is that almost all subjects were able to navigate the wheelchair quite well after a minimal training phase of no more than five minutes. Even if the average time of travel and the driven distance increased for test runs incorporating driving assistance, the number of collisions dropping to zero argues for this support, especially for untrained operators. The increase in travel time and driven distance is only significant in tight navigation situations as in our experiment. In situations without obstacles, both measures are not impeded by the assistance module. Hence, in normal situations the advantage of collision-free driving remains, while the drawbacks nearly disappear.

The experiments reported in this paper were conducted with healthy subjects. During the next months, the evaluation will be repeated with real users at the Fondazione Santa Lucia in Rome in the context of the SHARE-it project.

The Xeno with Driving Assistance was presented on the stand of Otto Bock Healthcare at the fair REHACARE 2008 in Germany (cf. Fig. 7). Spectators were allowed to test the wheelchair. For instance, they could drive the $66 \mathrm{~cm}$ wide vehicle through two $80 \mathrm{~cm}$ wide doorframes.

The wheelchair used as a basis for the work presented here, the Xeno, is a prototype model, because when we got it, the final version of the Xeno was not yet available on the market. Hence, parts of the casing are still missing. However, since the Xeno is available now (without our extensions) we will switch to the production model before we test the assistants with actual patients.

\section{REFERENCES}

[1] R. C. Simpson, "Smart wheelchairs: A literature review." Journal of Rehabilitation Research \& Development, no. 4, pp. 423-436.

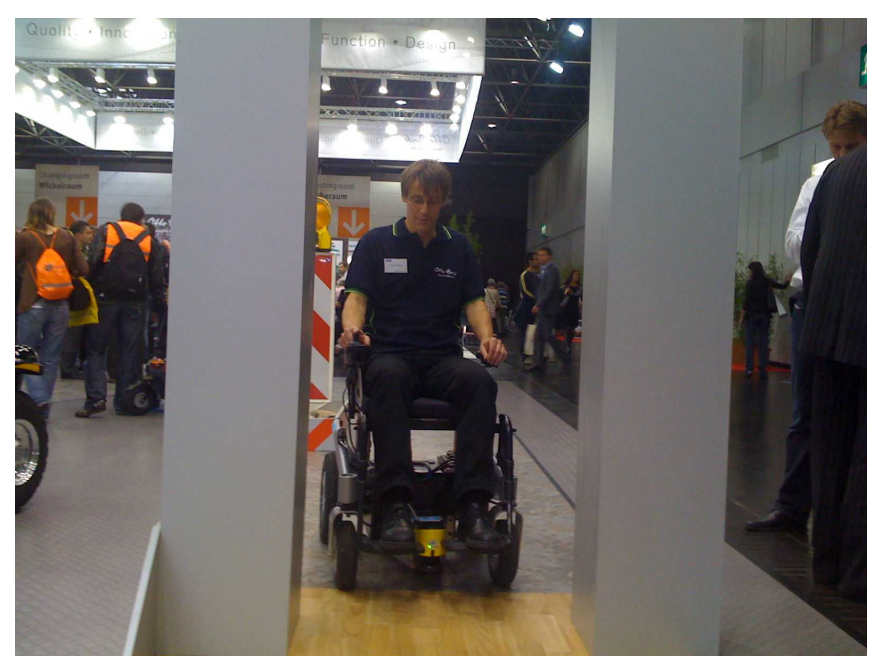

Fig. 7. The Driving Assistant at the fair REHACARE 2008 


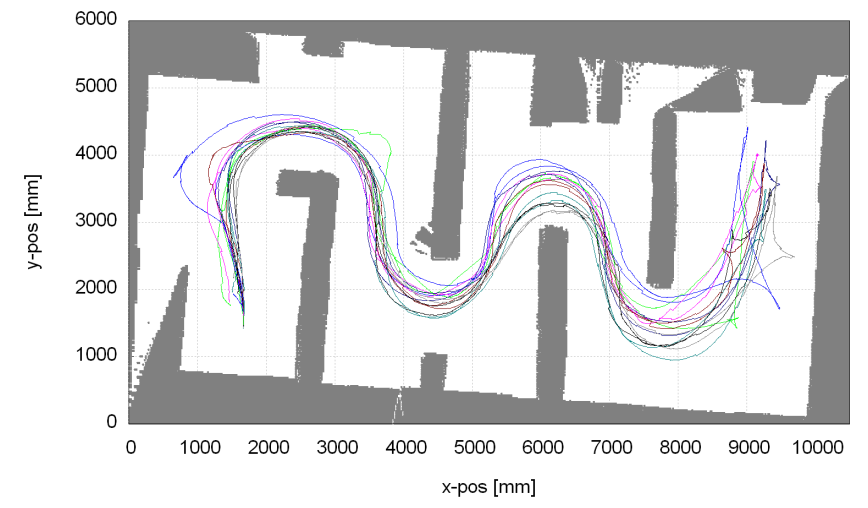

(a) Test run I: joystick without driving assistance

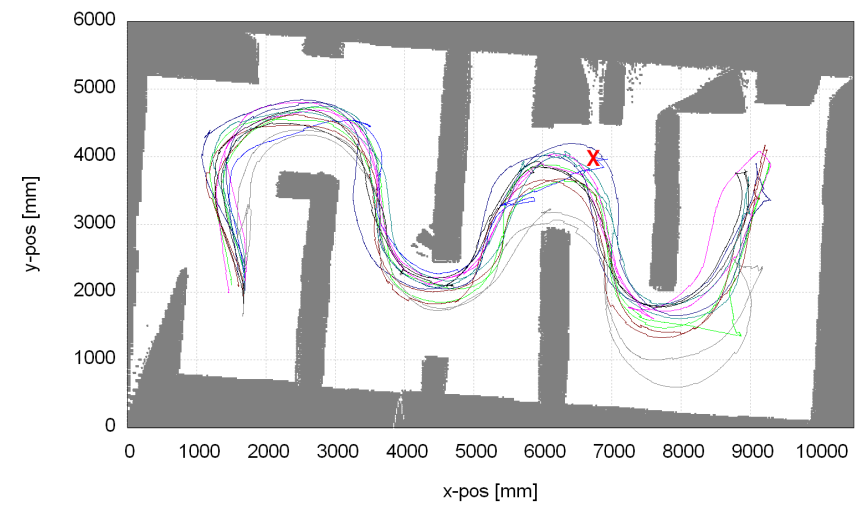

(c) Test run III: head-joystick without driving assistance

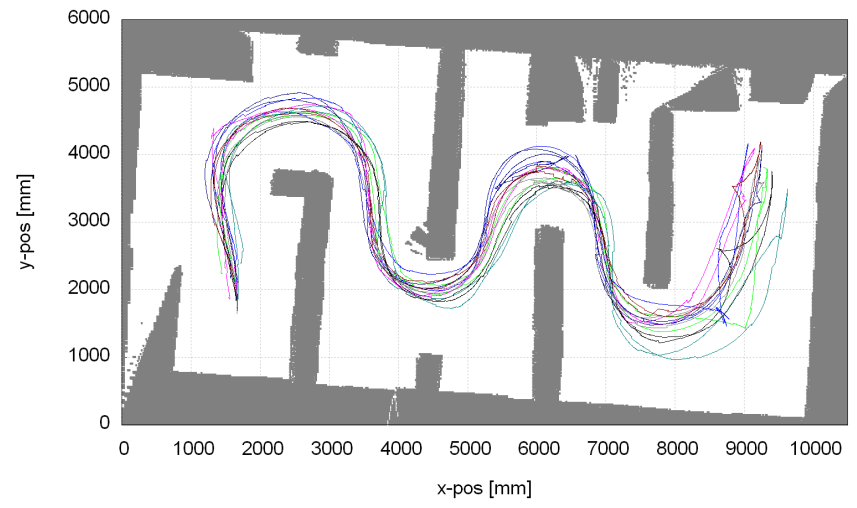

(b) Test run II: joystick with driving assistance

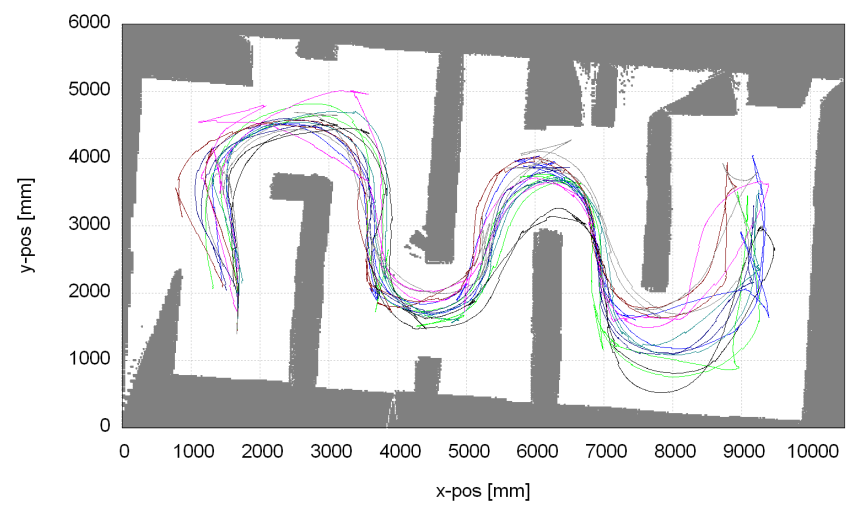

(d) Test run IV: head-joystick with driving assistance

Fig. 8. Plots of trajectories as driven by eight test persons within the scope of experimental test runs I-IV

[2] A. Lankenau and T. Röfer, "Smart wheelchairs - state of the art in an emerging market," Künstliche Intelligenz. Schwerpunkt Autonome Mobile Systeme, vol. 4, pp. 37-39, 2000.

[3] R. Simpson, S. Levine, D. Bell, L. Jaros, Y. Koren, and J. Borenstein, Lecture Notes in Computer Science: Assistive Technology and Artificial Intelligence. Springer Berling/Heidelberg, ch. NavChair: An assistive wheelchair navigation system with automatic adaption, pp. 235-255.

[4] R. Simpson and S. Levine, "Voice control of a powered wheelchair," IEEE Transactions on Neural Systems and Rehabilitation Engineering, vol. 10, no. 2, pp. 122-125, June 2002.

[5] A. Lankenau and T. Röfer, "A safe and versatile mobility assistant," Reinventing the Wheelchair. IEEE Robotics and Automation Magazine, no. 7, pp. 29-37, 2001.

[6] A. Elfes, "Occupancy grids: A stochastic spatial representation for active robot perception," in Autonomous Mobile Robots, S. S. Iyengar and A. Elfes, Eds., vol. 1. Los Alamitos, California: IEEE Computer Society Press, 1991, pp. 60-70.

[7] B. Rebsamen, E. Burdet, C. Guan, H. Zhang, C. Leong Teo, M. Ang, and C. Laugier, "Controlling a wheelchair using a BCI with low information transfer rate," in Proceedings of the 10th IEEE Intl. Conf. on Rehabilitation Robotics (ICORR), 2007.

[8] B. Graimann, B. Allison, C. Mandel, T. Lueth, D. Valbuena, and A. Gräser, Robust Intelligent Systems. Springer Verlag, 2007, ch. Noninvasive Brain-Computer Interfaces for Semi-Autonomous Assistive Devices, to appear.

[9] M. Bureau, J. Azkoitia, G. Ezmendi, I. Manterola, and H. Zabaleta, "Non-invasive, wireless and universal interface for the control of peripheral devices by means of head movements," in Proceedings of the 10th IEEE Intl. Conf. on Rehabilitation Robotics (ICORR), 2007.
[10] J. Gips, "On Building Intelligence into EagleEyes," in Assistive Technology and Artificial Intelligence, Applications in Robotics, User Interfaces and Natural Language Processing. Springer-Verlag, 1998, pp. 50-58.

[11] S. Levine, D. Bell, L. Jaros, R. Simpson, Y. Koren, and B. J., "The navchair assistive wheelchair navigation system," IEEE Transactions on Rehabilitation Engineering, vol. 7, no. 4, pp. 443-451, December 1999.

[12] C. Mandel, U. Frese, and T. Röfer, "Robot navigation based on the mapping of coarse qualitative route descriptions to route graphs," in Proceedings of the IEEE/RSJ International Conference on Intelligent Robots and Systems (IROS 2006), 2006, pp. 205-210.

[13] C. Mandel, T. Röfer, and U. Frese, "Applying a 3DOF orientation tracker as a human-robot interface for autonomous wheelchairs," in Proceedings of the 10th International Conference on Rehabilitation Robotics, 2007.

[14] C. Mandel, U. Frese, and T. Röfer, "Design improvements for proportional control of autonomous wheelchairs via $3 \mathrm{DOF}$ orientation tracker," in Proceedings of the 9th International Work-Conference on Artificial Neural Networks (IWANN'2007), ser. Lecture Notes in Computer Science. Springer; Berlin, 2007.

[15] C. Mandel and U. Frese, "Comparison of wheelchair user interfaces for the paralysed: Head-joystick vs. verbal path selection from an offered route-set," in Proceedings of the 3rd European Conference on Mobile Robots (ECMR 2007), 2007.

[16] “OpenSLAM web page," http://OpenSLAM.org/.

[17] G. Grisetti, C. Stachniss, and W. Burgard, "Improved techniques for grid mapping with rao-blackwellized particle filters," IEEE Transactions on Robotics, 2006. 